\title{
A Delay-dependent Condition for the Exponential Stability of Switched Linear Systems with Time-varying Delay
}

\author{
Kreangkri Ratchagit \\ Department of Mathematics, Faculty of Science \\ Maejo University, Chiang Mai, 50290, Thailand \\ E-mail: kreangkri@mju.ac.th
}

The research is financed by Faculty of Science, Maejo University.

\begin{abstract}
We use a Lyapunov-Krasovskii functional approach to establish the exponential stability of linear systems with timevarying delay. Our delay-dependent condition allows to compute simultaneously the two bounds that characterize the exponential stability rate of the solution. A simple procedure for constructing switching rule is also presented.
\end{abstract}

Keywords: Switched system, Time delay, Exponential stability, Lyapunov equation

\section{Introduction}

As an important class of hybrid systems, switched system is a family of differential equations together with rules to switch between them. A switched system can be described by a differential equation of the form

$$
\dot{x}=f_{\alpha}(t, x),
$$

where $\left\{f_{\alpha}():. \alpha \in \Omega\right\}$, is a family of functions that is parameterized by some index set $\Omega$, and $\alpha(\cdot) \in \Omega$ depending on the system state in each time is a switching rule/signal. The set $\Omega$ is typically a finite set. Switched systems arise in many practical models in manufacturing, communication networks, automotive engine control, chemical processes, and so on; see for example (D. Liberzon, 2003; A. V. Savkin, 2002; Z. Sun, 2005). During the previous decade, the stability problem of switched linear systems has attracted a lot of attention (see,; e.g. (D. Liberzon, 1999; V. N. Phat, 2006; Z. Sun, 2005) and the references therein). The main approach for stability analysis relies on the use of LyapunovKrasovskii functionals and LMI approach for constructing common Lyapunov function (R. N. Shorten, 1988). Although many important results have been obtained for switched linear systems, there are few results concerning the stability of the systems with time delay. Under commutative assumption on the system matrices, the authors of (K. S. Narendra, 1994) showed that when all subsystems are asymptotically stable, the switched system is asymptotically stable under arbitrary switching rule. The paper (G. Zhai, 2003) studied the asymptotic stability for switched linear systems with time delay, but the result was limited to symmetric systems. In (V. N. Phat, 2005; G. Xie, 2004), delay-dependent asymptotic stability conditions are extended to switched linear discrete-time linear systems with time delay. The exponential stability problem was considered in (Zairong Xi, 2003) for switched linear systems with impulsive effects by using matrix measure concept and in (G. D. Zong, 2004) for nonholonomic chained systems with strongly nonlinear input/state driven disturbances and drifts. In recent paper (S. Kim, 2006), studying a switching system composed of a finite number of linear delay differential equations, it was shown that the asymptotic stability of this kind of switching systems may be achieved by using a common Lyapunov function method switching rule. There are some other results concerning asymptotic stability for switched linear systems with time delay, but we do not find any result on exponential stability even for the switched systems without delay except (Zairong Xi, 2003; G. D. Zong, 2004). On the other hand, it is worth noting that the existing stability conditions for time-delay systems must be solved upon a grid on the parameter space, which results in testing a nonlinear Riccati-like equation or a finite number of LMIs. In this case, the results using finite gridding points are unreliable and the numerical complexity of the tests grows rapidly. Therefore, finding simple stability conditions for switched linear systems with time-delay is of interest.

In this paper, we study the exponential stability of a class of switched linear systems with time-varying delay. The system studied in this paper is time-varying under a switching rule dependent on continuous system states. A delay-dependent condition for the exponential stability are formulated in terms of a generalized Lyapunov equation for linear systems with time-varying delay, which allows easily to compute simultaneously the two bounds that characterize the exponential stability rate of the solution. A simple procedure for constructing switching rule is presented. The results obtained in this paper can be partly considered as extensions of existing results for linear time-delay systems and for switched linear systems without time delays.

The organization of this paper is as follows. Following the introduction and the problem motivation, Section 2 presents the notation, definitions and auxiliary propositions. The main result is given in Section 3 and followed by an numerical example and conclusion. 


\section{Problem formulation}

The following notations will be used throughout this paper. $\mathbb{R}^{+}$denotes the set of all non-negative real numbers; $\mathbb{R}^{n}$ denotes the $n$-finite-dimensional Euclidean space, with the Euclidean norm $\|$.$\| and scalar product x^{T} y$ of two vectors $x, y$. $\mathbb{R}^{n \times m}$ denotes the set of all $(n \times m)$-matrices; $\lambda_{\max }(A)\left(\lambda_{\min }(A)\right.$, resp.) denotes the maximal number (the minimum number, resp.) of the real part of eigenvalues of $A ; A^{T}$ denotes the transpose of the matrix $A ; Q \geq 0(Q>0$, resp.) means $Q$ is semi-positive definite (positive definite, resp.).

Consider a switched linear system with time-varying delay of the form

$$
\Sigma_{\alpha} \quad\left\{\begin{array}{l}
\dot{x}(t)=A_{\alpha} x(t)+D_{\alpha} x(t-h(t)), \quad t \in \mathbb{R}^{+}, \\
x(t)=\phi(t), \quad t \in[-h, 0],
\end{array}\right.
$$

where $x(t) \in \mathbb{R}^{n}$ is the continuous trajectory of system, $A_{\alpha}, D_{\alpha} \in \mathbb{R}^{n \times n}$ are given constant matrices, $\phi(t) \in C\left([-h, 0], \mathbb{R}^{n}\right)$ is the initial function with the norm $\|\phi\|=\sup _{s \in[-h, 0]}\|\phi(s)\| . \alpha(x): \mathbb{R}^{n} \rightarrow \Omega:=\{1,2, \ldots, N\}$ is the switching rule, which is a piece-wise constant function depending on the state in each time. A switching rule is a rule which determines a switching sequence for a given switching system. Moreover, $\alpha(x)=i$ implies that the system realization is chosen as $\left[A_{i}, B_{i}\right], i=1,2, \ldots, N$. It is seen that the system (1) can be viewed as an linear autonomous switched system in which the effective subsystem changes when the state $x(t)$ hits predefined boundaries; i.e., the switching rule is dependent on the system trajectory. The time-varying delay function $h(t)$ satisfies the following assumption

$$
0 \leq h(t) \leq h, \quad h>0, \quad \dot{h}(t) \leq \mu<1 .
$$

This assumption means that the time delay may change from time to time, but the rate of changing is bounded. Also, due to the upper bound, the delay can not increase as fast as the time itself. In fact, the function $h(t)$ can be different for each subsystem; i.e., it should be denoted by $h_{i}(t)$, but we assume $h_{i}(t)$ be the same value in this paper for convenient formulation.

The exponential stability problem for switched linear system (1) is to construct a switching rule that makes the system exponentially stable.

Definition 2.1 Switched linear system (1) is exponentially stable if there exists switching rule $\alpha($.) such that every solution $x(t, \phi)$ of the system $\Sigma_{\alpha}$ satisfies the condition

$$
\exists M>0, \delta>0:\|x(t, \phi)\| \leq M e^{-\delta t}\|\phi\|, \quad \forall t \in \mathbb{R}^{+} .
$$

The numbers $N>0$ and $\delta>0$ are called the stability factor and decay rate of the system.

Definition 2.2 (D. P. Stanford, 1979) System of symmetric matrices $\left\{L_{i}\right\}, i=1,2, \ldots, N$, is said to be strictly complete if for every $0 \neq x \in \mathbb{R}^{n}$ there is $i \in\{1,2, \ldots, N\}$ such that $x^{T} L_{i} x<0$.

Let us define the sets

$$
\Omega_{i}=\left\{x \in \mathbb{R}^{n}: x^{T} L_{i} x<0\right\}, \quad i=1,2, \ldots, N .
$$

It is easy to show that the system $\left\{L_{i}\right\}, i=1,2, \ldots, N$, is strictly complete if and only if $\cup_{i=1}^{N} \Omega_{i}=\mathbb{R}^{n} \backslash\{0\}$.

Remark 2.3 As shown in (F. Uhlig, 1979), a sufficient condition for the strict completeness of the system $\left\{L_{i}\right\}$ is that there exist numbers $\tau_{i} \geq 0, i=1,2, \ldots, N$, such that $\sum_{i=1}^{N} \tau_{i}>0$ and

$$
\sum_{i=1}^{N} \tau_{i} L_{i}<0
$$

and in the case if $N=2$, then the above condition is also necessary for the strict completeness.

Before presenting the main result, we recall the following well-known matrix inequality and Lyapunov stability theorem for time delay systems.

Proposition 2.4 For any $\epsilon>0, x, y \in \mathbb{R}^{n}$, we have

$$
-2 x^{T} y \leq \epsilon^{-1} x^{T} x+\epsilon y^{T} y .
$$

Proposition $2.5\left(\mathrm{~J}\right.$. K. Hale, 1993) Let $x_{t}:=\{x(t+s), s \in[-h, 0]\}$. Consider nonlinear delay system

$$
\begin{gathered}
\dot{x}(t)=f\left(t, x_{t}\right), \\
x(t)=\phi(t), \\
f(t, 0)=0, t \in \mathbb{R}^{+} .
\end{gathered}
$$

If there exists a Lyapunov function $V\left(t, x_{t}\right)$ satisfying the following conditions: 
(i) There exists $\lambda_{1}>0, \lambda_{2}>0$ such that $\lambda_{1}\|x(t)\|^{2} \leq V\left(t, x_{t}\right) \leq \lambda_{2}\left\|x_{t}\right\|^{2}$, for all $t \in \mathbb{R}^{+}$,

(ii) $\dot{V}_{f}\left(t, x_{t}\right):=\frac{\partial V}{\partial t}+\frac{\partial V}{\partial x} f\left(t, x_{t}\right) \leq 0$, for all solutions $x(t)$ of the system,

then the solution $x(t, \phi)$ is bounded: There exists $N>0$ such that $\|x(t, \phi)\| \leq N\|\phi\|$, for all $t \in \mathbb{R}^{+}$.

\section{Main result}

For given positive numbers $\mu, \delta, h, \epsilon$ we set

$$
\begin{gathered}
\beta=(1-\mu)^{-1}, \\
L_{i}(P)=A_{i}^{T} P+P A_{i}+\epsilon^{-1} e^{2 \delta h} D_{i}^{T} P D_{i}+(\epsilon \beta+2 \delta) P . \\
S_{i}^{P}=\left\{x \in \mathbb{R}^{n}: \quad x^{T} L_{i}(P) x<0\right\}, \\
\bar{S}_{1}^{P}=S_{1}^{P}, \quad \bar{S}_{i}^{P}=S_{i}^{P} \backslash \bigcup_{j=1}^{i-1} \bar{S}_{j}^{P}, \quad i=2,3, \ldots, N, \\
\lambda_{\max }(D)=\max _{i=1,2, \ldots, N} \lambda_{\max }\left(D_{i}^{T} D_{i}\right), \\
M=\sqrt{\frac{\lambda_{\max }(P)}{\lambda_{\min }(P)}+\frac{\epsilon^{-1}\left(e^{2 \delta h}-1\right) \lambda_{\max }(P) \lambda_{\max }(D)}{2 \delta}} .
\end{gathered}
$$

The main result of this paper is summarized in the following theorem.

Theorem 3.1 Switched linear system (1) is exponentially stable if there exist positive numbers $\epsilon, \delta$ and symmetric positive definite matrix $P \in \mathbb{R}^{n \times n}$ such that one of the following conditions holds

(i) The system of matrices $\left\{L_{i}(P)\right\}, i=1,2, \ldots, N$, is strictly complete.

(ii) There exists $\tau_{i} \geq 0, i=1,2, \ldots, N$, with $\sum_{i=1}^{N} \tau_{i}>0$ and

$$
\sum_{i=1}^{N} \tau_{i} L_{i}(P)<0
$$

The switching rule is chosen in case $(i)$ as $\alpha(x(t))=i$ whenever $x(t) \in \bar{S}_{i}$, and in case (ii) as

$$
\alpha(x(t))=\arg \min \left\{x^{T}(t) L_{i}(P) x(t)\right\} .
$$

Moreover, the solution $x(t, \phi)$ of the system satisfies

$$
\|x(t, \phi)\| \leq M e^{-\delta t}\|\phi\|, \quad t \in \mathbb{R}^{+} .
$$

In the case $N=2$, the conditions ( $i$ ) and (ii) are equivalent.

Proof. For $\delta>0$, we utilize the following state transformation $y(t)=e^{\delta t} x(t)$. The system (1) is transformed into

$$
\begin{gathered}
\dot{y}(t)=\bar{A}_{\alpha} y(t)+e^{\delta h(t)} D_{\alpha} y(t-h(t)), t \in \mathbb{R}^{+}, \\
y(t)=e^{\delta t} \phi(t), \quad t \in[-h, 0],
\end{gathered}
$$

where $\bar{A}_{\alpha}:=A_{\alpha}+\delta I$. Assume that the condition (i) of the theorem holds. For every $i=1,2, \ldots, N$, we consider the Lyapunov-Krasovskii functional

$$
V\left(t, y_{t}\right)=\langle P y(t), y(t)\rangle+\epsilon^{-1} e^{2 \delta h} \int_{t-h(t)}^{t}\left\langle D_{i}^{T} P D_{i} y(s), y(s)\right\rangle d s
$$

It is easy to see that there are positive numbers $\lambda_{1}, \lambda_{2}$ such that

$$
\lambda_{1}\|y(t)\|^{2} \leq V\left(t, y_{t}\right) \leq \lambda_{2}\left\|y_{t}\right\|^{2}, \quad \forall t \in \mathbb{R}^{+} .
$$


The derivative along the trajectory of the system (3) is

$$
\begin{aligned}
\dot{V}\left(t, y_{t}\right)= & 2\langle P \dot{y}(t), y(t)\rangle+\epsilon^{-1} e^{2 \delta h}\left\langle D_{i}^{T} P D_{i} y(t), y(t)\right\rangle \\
& -\epsilon^{-1} e^{\delta h}(1-\dot{h}(t))\left\langle D_{i}^{T} P D_{i} y(t-h(t)), y(t-h(t))\right\rangle \\
\leq & \left\langle\left(A_{i}^{T} P+P A_{i}+2 \delta P+\epsilon^{-1} e^{\delta h} D_{i}^{T} P D_{i}\right) y(t), y(t)\right\rangle \\
& +2 e^{2 \delta h(t)}\left\langle P D_{i} y(t-h(t)), y(t)\right\rangle \\
& -\epsilon^{-1} e^{\delta h}(1-\mu)\left\langle D_{i}^{T} P D_{i} y(t-h(t)), y(t-h(t))\right\rangle .
\end{aligned}
$$

Since $P$ is a symmetric positive definite matrix, there is $\bar{P}=P^{1 / 2}$ such that $P=\bar{P}^{T} \bar{P}$. Using Proposition 2.1 , for any $\xi>0$, we have

$$
\begin{aligned}
& 2 e^{\delta h(t)}\left\langle P D_{i} y(t-h(t)), y(t)\right\rangle \\
& =2 y^{T}(t) \bar{P}^{T} \bar{P} e^{\delta h(t)} D_{i} y(t-h(t)) \\
& \leq \xi y^{T}(t) \bar{P}^{T} \bar{P} y(t)+\xi^{-1} e^{2 \delta h(t)} y^{T}(t-h(t)) D_{i}^{T} \bar{P}^{T} \bar{P} D_{i} y(t-h(t)) .
\end{aligned}
$$

Taking $\xi=\epsilon \beta>0$, and since $h(t) \leq h$, we obtain

$$
\begin{aligned}
& 2 e^{\delta h(t)}\left\langle P D_{i} y(t-h(t)), y(t)\right\rangle \\
& \leq \epsilon \beta y^{T}(t) P y(t)+\epsilon^{-1}(1-\mu) e^{2 \delta h} y^{T}(t-h(t)) D_{i}^{T} P D_{i} y(t-h(t)) .
\end{aligned}
$$

Then from (5) it follows that

$$
\begin{aligned}
\left.\dot{V}\left(t, y_{t}\right)\right) & \leq\left\langle\left(A_{i}^{T} P+P A_{i}+\epsilon^{-1} e^{2 \delta h} D_{i}^{T} P D_{i}+(\epsilon \beta+2 \delta) P\right) y(t), y(t)\right\rangle \\
& =y^{T}(t) L_{i}(P) y(t) .
\end{aligned}
$$

By the assumption, the system of matrices $\left\{L_{i}(P)\right\}$ is strictly complete. We have

$$
\bigcup_{i=1}^{N} S_{i}^{P}=\mathbb{R}^{n} \backslash\{0\} .
$$

Based on the sets $S_{i}^{P}$ we constructing the sets $\bar{S}_{i}^{P}$ as above and we can verify that

$$
\bar{S}_{i}^{P} \bigcap \bar{S}_{j}^{P}=\{0\}, i \neq j, \quad \bar{S}_{i}^{P} \bigcup \bar{S}_{j}^{P}=\mathbb{R}^{n} \backslash\{0\} .
$$

We then construct the following switching rule: $\alpha(x(t))=i$, whenever $x(t) \in \bar{S}_{i}^{P}$ (this switching rule is well-defined due to the condition (8)). From the state transformation $y(t)=e^{\delta t} x(t)$ and taking (6) into account we obtain

$$
\dot{V}\left(t, y_{t}\right)=y^{T}(t) L_{\alpha}(P) y(t)=e^{2 \delta t} x^{T}(t) L_{\alpha}(P) x(t) \leq 0, \quad \forall t \in \mathbb{R}^{+},
$$

which implies that the solution $y(t)$ of system (3), by Proposition 2.2, is bounded. Returning to the state transformation of $y(t)=e^{\delta t} x(t)$ guarantees the exponentially stability with the decay rate $\delta$ of the system (1). To define the stability factor $M$, integrating both sides of (9) from 0 to $t$ and using the expression of $V\left(t, y_{t}\right)$ from (4) we have

$$
\langle P y(t), y(t)\rangle+\epsilon^{-1} e^{\delta h} \int_{t-h(t)}^{t}\left\langle D_{i}^{T} P D_{i} y(s), y(s)\right\rangle d s \leq V(y(0)) .
$$

Since $D_{i}^{T} P D_{i}>0$, and

$$
\lambda_{\min }\|y(t)\|^{2} \leq\langle P y(t), y(t)\rangle
$$

we have

$$
\lambda_{\min }\|y(t)\|^{2} \leq \lambda_{\max }(P)\|y(0)\|^{2}+\epsilon^{-1} e^{2 \delta h} \int_{h(0)}^{0}\left\langle D_{i}^{T} P D_{i} y(s), y(s)\right\rangle d s .
$$

We have

$$
\begin{aligned}
\int_{-h(0)}^{0}\left\langle D_{i}^{T} P D_{i} y(s), y(s)\right\rangle d s & \leq \lambda_{\max }(P) \lambda_{\max }(D)\|\phi\|^{2} \int_{-h(0)}^{0} e^{2 \delta s} d s \\
& =\frac{\lambda_{\max }(P) \lambda_{\max }(D)}{2 \delta}\left(1-e^{-2 \delta h(0)}\right)\|\phi\|^{2} \\
& \leq \frac{\lambda_{\max }(P) \lambda_{\max }(D)}{2 \delta}\left(1-e^{-2 \delta h}\right)\|\phi\|^{2}
\end{aligned}
$$


we have

$$
\|y(t)\|^{2} \leq\left[\frac{\lambda_{\max }(P)}{\lambda_{\min }(P)}+\frac{\epsilon^{-1} e^{2 \delta h} \lambda_{\max }(P) \lambda_{\max }(D)}{2 \delta}\left(1-e^{-2 \delta h}\right)\right]\|\phi\|^{2} .
$$

Therefore,

$$
\|y(t)\| \leq M\|\phi\|, \quad \forall t \in \mathbb{R}^{+},
$$

where

$$
M=\sqrt{\frac{\lambda_{\max }(P)}{\lambda_{\min }(P)}+\frac{\epsilon^{-1}\left(e^{2 \delta h}-1\right) \lambda_{\max }(P) \lambda_{\max }(D)}{2 \delta}} .
$$

We now assume the condition (ii), then we have

$$
\sum_{i=1}^{N} \tau_{i} L_{i}(P)<0
$$

where $\tau_{i} \geq 0, i=1,2, \ldots, N, \sum_{i=1}^{N} \tau_{i}>0$. Since the numbers $\tau_{i}$ are non-negative and $\sum_{i=1}^{N} \tau_{i}>0$, there is always a number $\epsilon>0$ such that for any nonzero $y(t)$ we have

$$
\sum_{i=1}^{N} \tau_{i} y^{T}(t) L_{i}(P) y(t) \leq-\epsilon y^{T}(t) y(t)
$$

Therefore,

$$
\sum_{i=1}^{N} \tau_{i} \min _{i=1, \ldots, N}\left\{y^{T}(t) L_{i}(P) y(t)\right\} \leq \sum_{i=1}^{N} \tau_{i} y^{T}(t) L_{i}(P) y(t) \leq-\epsilon y^{T}(t) y(t) .
$$

The Lyapunov-Krasovskii functional $V($.$) is defined by (4) and the switching rule is designed as follows$

$$
\alpha(x(t))=\arg \min _{i=1, \ldots, N}\left\{x^{T}(t) L_{i}(P) x(t)\right\} .
$$

Combining (6) and (10) gives

$$
\left.\dot{V}\left(t, y_{t}\right)\right) \leq-\eta\|y(t)\|^{2} \leq 0, \quad \forall t \in \mathbb{R}^{+},
$$

where $\eta=\epsilon\left(\sum_{i=1}^{N} \tau_{i}\right)^{-1}$. This implies that all the solution $y(t)$ of system (3) are bounded. The proof is then completed by the same way as in the part (i).

Remark 3.2 Note that conditions (i) and (ii) involve linear Lyapunov-type matrix inequality, which is easy to solve. The following simple procedure can be applied to construct switching rule and define the stability factor and decay rate of the system.

Step 1. Define the matrices $L_{i}(P)$.

Step 2. Find the solution $P$ of the generalized Lyapunov inequality (2).

Step 3. Construct the sets $S_{i}^{P}$, and then $\bar{S}_{i}^{P}$, and verify the condition (7), (8).

Step 4. The switching signal $\alpha\left(\right.$.) is chosen as $\alpha(x)=i$, whenever $x \in \bar{S}_{i}^{P}$ or as

$$
\alpha(x(t))=\arg \min \left\{x^{T}(t) L_{i}(P) x(t)\right\} .
$$

Example 3.3 Consider the switched linear system defined by

$$
\dot{x}(t)=A_{i} x(t)+D_{i} x(t-h(t)), \quad i=1,2
$$

where

$$
\begin{gathered}
\left(A_{1}, D_{1}\right)=\left(\left[\begin{array}{cc}
0.39 & 0.09 \\
0.09 & -1.49
\end{array}\right],\left[\begin{array}{cc}
0.39 & 0.03 \\
0.03 & -0.29
\end{array}\right]\right), \\
\left(A_{2}, D_{2}\right)=\left(\left[\begin{array}{cc}
-1.99 & -0.13 \\
-0.13 & 0.59
\end{array}\right],\left[\begin{array}{cc}
-0.09 & -0.01 \\
-0.01 & 0.19
\end{array}\right]\right), \\
h(t)=\sin ^{2}(0.1 t) .
\end{gathered}
$$

We have $h=1, \dot{h}(t)=0.1 \sin (0.2 t)$ and then $\mu=0.1$. By choosing the positive definite matrix $P>0$ as

$$
P=\left[\begin{array}{cc}
1 & 0.1 \\
0.1 & 1
\end{array}\right]
$$


and $\epsilon=0.1, \delta=0.001$, one can verify condition (2) with $\tau_{1}=\tau_{2}=0.5$. The switching regions are given as

$$
\begin{aligned}
& \bar{S}_{1}=\left\{\left(x_{1}, x_{2}\right): 2.468 x_{1}^{2}-0.002 x_{1} x_{2}-2.015 x_{2}^{2}<0\right\}, \\
& \bar{S}_{2}=\left\{\left(x_{1}, x_{2}\right): 2.468 x_{1}^{2}-0.002 x_{1} x_{2}-2.015 x_{2}^{2}>0\right\} .
\end{aligned}
$$

According to Theorem 3.1, the system with the switching rule $\alpha(x(t))=i$ if $x(t) \in \bar{S}_{i}$ is exponentially stable. In this case, it can be checked that

$$
\left(L_{1}(P), L_{2}(P)\right)=\left(\left[\begin{array}{cc}
2.468 & -0.001 \\
-0.001 & -2.015
\end{array}\right],\left[\begin{array}{cc}
-3.809 & -0.416 \\
-0.416 & 1.626
\end{array}\right]\right) .
$$

Moreover, the sum

$$
\tau_{1} L_{1}(P)+\tau_{2} L_{2}(P)=\left[\begin{array}{ll}
-0.6705 & -0.2085 \\
-0.2085 & -0.1945
\end{array}\right]
$$

is negative definite; i.e. the first entry in the first row and the first column $-0.6705<0$ is negative and the determinant of the matrix is positive. The sets $S_{1}$ and $S_{2}$ (without bar) are given as

$$
\begin{gathered}
S_{1}=\left\{\left(x_{1}, x_{2}\right): 2.468 x_{1}^{2}-0.002 x_{1} x_{2}-2.015 x_{2}^{2}<0\right\}, \\
S_{2}=\left\{\left(x_{1}, x_{2}\right):-3.809 x_{1}^{2}-0.831 x_{1} x_{2}+1.626 x_{2}^{2}<0\right\} .
\end{gathered}
$$

These sets are equivalent to

$$
S_{1}=\left\{\left(x_{1}, x_{2}\right):\left(x_{2}+1.107 x_{1}\right)\left(x_{2}-1.106 x_{1}\right)>0\right\},
$$

and

$$
S_{2}=\left\{\left(x_{1}, x_{2}\right):\left(x_{2}+1.296 x_{1}\right)\left(x_{2}-1.807 x_{1}\right)<0\right\} ;
$$

see figure 1 . Obviously, the union of these sets is equal to $\mathbb{R}^{2}$.

Example 3.4 Consider switched linear uncertain delay system (1) with any initial function $\phi(t)$, where $N=2, h(t)=$ $\sin ^{2} 0.5 t$, and

$$
\begin{aligned}
& A_{1}=\left(\begin{array}{cc}
a_{1} & 0 \\
0 & a_{2}
\end{array}\right), \quad A_{2}=\left(\begin{array}{cc}
a_{3} & 0 \\
0 & a_{4}
\end{array}\right), \\
& D_{1}=\left(\begin{array}{ll}
1 & 1 \\
1 & 1
\end{array}\right), \quad D_{2}=\left(\begin{array}{cc}
e^{-2} & e^{2} \\
e^{2} & e^{-2}
\end{array}\right),
\end{aligned}
$$

where

$$
\begin{gathered}
a_{1}=-1.875 e^{4}-3, \quad a_{2}=-3.75 e^{4}-3, \\
a_{3}=-0.125 e^{8}-0.3125 e^{4}-3.5, \quad a_{4}=-2 e^{8}-5 e^{4}-3.5 .
\end{gathered}
$$

We have $h=1$, and since $\dot{h}(t)=0.5 \sin t, \mu=0.5, \beta=2$. Taking $\epsilon=1, \delta=2$ and

$$
P=\left(\begin{array}{cc}
0.4 e^{-4} & 0 \\
0 & 0.1 e^{-4}
\end{array}\right)
$$

we find

$$
L_{1}(P)=\left(\begin{array}{cc}
-1 & 0.5 \\
0.5 & -0.25
\end{array}\right), \quad L_{2}(P)=\left(\begin{array}{cc}
-0.25 & 0.5 \\
0.5 & -1
\end{array}\right)
$$

Therefore,

$$
\begin{aligned}
& S_{1}^{P}=\left\{\left(x_{1}, x_{2}\right) \in \mathbb{R}^{2}: x_{1} \neq 0.5 x_{2}\right\}, \\
& S_{2}^{P}=\left\{\left(x_{1}, x_{2}\right) \in \mathbb{R}^{2}: x_{2} \neq 0.5 x_{1}\right\} .
\end{aligned}
$$

So

Therefore, taking

$$
S_{1}^{P} \bigcup S_{2}^{P}=\mathbb{R}^{2} \backslash\{0\}
$$

$$
\overline{\boldsymbol{S}}_{1}^{P}=\boldsymbol{S}_{1}^{P} \quad \overline{\boldsymbol{S}}_{2}^{P}=\left\{\left(x_{1}, x_{2}\right) \in \mathbb{R}^{2}: \quad x_{1}=0.5 x_{2}, x_{1} \neq 0, x_{2} \neq 0\right\},
$$

we have

$$
\overline{\boldsymbol{S}}_{1}^{P} \cup \overline{\boldsymbol{S}}_{2}^{P}=\mathbb{R}^{2} \backslash\{0\}, \quad \overline{\boldsymbol{S}}_{1}^{P} \cap \overline{\boldsymbol{S}}_{2}^{P}=\emptyset .
$$


Then, the switching signal is chosen as

$$
\alpha(x)= \begin{cases}1, & \text { if } x \in \overline{\boldsymbol{S}}_{1}^{P}, \\ 2, & \text { if } x \in \overline{\boldsymbol{S}}_{2}^{P}\end{cases}
$$

To find the exponent factor $M$, computing

$$
\begin{aligned}
\lambda_{\max }(P) & =0.4 e^{-4}, \quad \lambda_{\min }(P)=0.1 e^{-4}, \\
\lambda_{\max }\left(D_{1}^{T} D_{1}\right) & =4 \quad \lambda_{\max }\left(D_{2}^{T} D_{2}\right)=e^{4}+e^{-4}+2,
\end{aligned}
$$

we have $M \approx 3.085$. Therefore, the solution of the system satisfies the inequality

$$
\|x(t, \phi)\| \leq 3.085\|\phi\| e^{-2 t}, \quad \forall t \geq 0 .
$$

\section{Conclusions}

We have presented a delay-dependent condition for the exponential stability of switched linear systems with time-varying delay. The condition is formulated in terms of a generalized Lyapunov equation, which allows easily to compute simultaneously the two bounds that characterize the exponential stability rate of the solution. A simple procedure for constructing switching rule has been given.

\section{Acknowledgments}

This paper was supported by the Basic Program in Natural Sciences, Thailand Research Fund Grant. The authors would like to thank anonymous referees for his/her valuable comments and remarks that improved the paper.

\section{References}

A. V. Savkin and R. J. Evans. (2002). Hybrid Dynamical Systems : Controller and Sensor Switching Problems. Boston: Birkhauser.

C. Wang, L. X. Zhang, H. J. Gao and L. G. Wu. (2005). Delay-dependent stability and stabilization of a class of linear switched time-varying delay systems. Proc. IEEE Mech. Learning Cyber., Guangzhou, August, 917-922.

D. Liberzon. (2003). Switching in Systems and Control. Boston, MA: Birkhauser.

D. Liberzon and A. S. Morse. (1999). Basic problems in stability and design of switched systems. IEEE Control Systems Magazine, 19(5): 59-69.

D. P. Stanford. (1979). Stability for a multi-rate sampled-data system. SIAM J. Contr. Optim., 17, 390-399.

F. Uhlig. (1979). A recurring theorem about pairs of quadratic forms and extensions. Linear Algebra Appl., 25, $219-237$.

G. D. Zong and Y. Q. Wu. (2004). Exponential stability of a class of switched and hybrid systems. Proc. IEEE on Contr. Aut. Robotics and Vision, Kuming, China, December, 2244-2249.

G. Xie and L. Wang. (2004). Quadratic stability and stabilization of discrete-time switched systems with state delay. Proc. IEEE Confer. on Decision and Control, Atlantis, December, 3235-3240.

G. Zhai, Y. Sun, X. Chen and A. Michel. (2003). Stability and $L_{2}$ gain analysis for switched symmetric systems with time delay. Proc. Amer. Contr. Confere., Colorado, USA, June, 2682-2687.

J. K. Hale and S. M. Lunel. (1993). Introduction to Functional Differential Equation. New York, Springer.

K. S. Narendra and V. Balakrishnan. (1994). A common Lyapunov function for stable LTI systems with commuting matrices. IEEE Trans. AC, 39, 2469-2471.

R. N. Shorten and K. S. Narendra. (1988). On the stability and existence of common Lyapunov functions for stable swithching systems Proc. of the 37th IEEE Conf. on DEFC, Florida, 12: 3723-3724.

S. Kim, S. A. Campbell and X. Liu. (2006). Stability of a class of linear switching systems with time-delay. IEEE Trans. Circ. and Syst., II, 53, 384-393.

V. N. Phat. (2005). Robust stability and stabilizability of uncertain linear hybrid systems with state delays. IEEE Trans. on CAS II, 52(1): 94-98.

V. N. Phat and S. Pairote. (2006). Global stabilization of linear periodically time-varying switched systems via matrix inequalities. J. Contr. Theory Appl., 1, 26-31.

Zairong Xi, G. Feng, Z. P. Jiang and D. Cheng. (2003). A switching algorithm for global expopnential stabilization of uncertain chained systems. IEEE Trans. Aut. Contr., 48, 1793-1798.

Z. Sun and S. S. Ge. (2005). Analysis and synthesis of switched linear control systems. Automatica, 41, 181-195.

Z. Sun and S. S. Ge. (2005). Switched Linear Systems: Control and Design. Springer, London. 